\title{
The non-triviality of the Grope filtrations of the knot and link concordance groups
}

Peter D. Horn*

\begin{abstract}
We consider the Grope filtration of the classical knot concordance group that was introduced by Cochran, Orr and Teichner. Our main result is that each successive quotient in this filtration has infinite rank. We also establish the analogous result for the Grope filtration of the concordance group of string links consisting of more than one component.
\end{abstract}

Mathematics Subject Classification (2010). Primary 57M25; Secondary 57N70.

Keywords. Concordance, Grope filtration, solvable filtration.

\section{Introduction}

We work in the smooth category. A knot is an oriented, smooth embedding of the circle in the three-sphere. Two knots are isotopic if there is a smooth isotopy of the three-sphere taking one knot to the other. Isotopy is a three-dimensional notion of knot equivalence. In this paper, we study a four-dimensional notion of knot equivalence called concordance. Two knots $K_{0}$ and $K_{1}$ are concordant if there exists a smooth embedding of an annulus $A=S^{1} \times[0,1]$ into $S^{3} \times[0,1]$ such that $A \cap\left(S^{3} \times\{i\}\right)=K_{i}$ for $i=0,1$. One can check that isotopic knots are concordant, and one can find counterexamples to the converse. Given two knots, one can combine them via the connect sum operation, denoted \#. See [Rol76, p. 39] for more details. The set of knots modulo concordance, equipped with the connect sum operation, forms an abelian group. We wish to study this concordance group $e$.

In [COT03], Cochran, Orr, and Teichner introduced two filtrations of the knot concordance group by subgroups. The $(n)$-solvable filtration

$$
\cdots \subset \mathscr{F}_{n .5} \subset \mathscr{F}_{n} \subset \cdots \subset \mathscr{F}_{1} \subset \mathscr{F}_{0.5} \subset \mathscr{F}_{0} \subset \mathcal{Y}
$$

is defined by $K \in \mathcal{F}_{n}$ if $M_{K}$, the closed 3-manifold obtained by zero-framed surgery on $S^{3}$ along $K$, bounds a 4-manifold $W$ whose intersection form on $\mathrm{H}_{2}$ is a direct

\footnotetext{
* The author was partially supported by National Science Foundation Grant DMS 0240058.
} 
sum of hyperbolic pairs, even when homology is considered with twisted coefficients coming from the quotient of $\pi_{1}(W)$ by its $n$-th derived subgroup. Precise definitions will be provided in Section 2. A slice knot lies in $\mathscr{F}_{n}$ for all $n \in \frac{1}{2} \mathbb{N}$. In this light, the maximum $n$ for which $K \in \mathscr{F}_{n}$ but $K \notin \mathscr{F}_{n .5}$ is a measure of how close $K$ is to being a slice knot.

The other, more geometric filtration defined in [COT03] is the Grope filtration

$$
\cdots \subset \mathscr{E}_{n .5} \subset \mathscr{E}_{n} \subset \cdots \subset \mathscr{E}_{3} \subset \mathscr{E}_{2.5} \subset \mathscr{E}_{2} \subset \mathcal{C}
$$

which is defined by $K \in \mathscr{E}_{n}$ if $K$ bounds a Grope of height $n$ in $D^{4}$. A grope is a 2-complex that will be defined in Section 2. As in the $(n)$-solvable filtration, a slice knot lies in $\mathscr{E}_{n}$ for all $n$, and so the largest $n$ for which $K \in \mathscr{E}_{n}$ but $K \notin \mathscr{E}_{n .5}$ is some measure of how far $K$ is from being slice.

The non-triviality of the $(n)$-solvable filtration has been a topic of study since its introduction. That $\mathscr{F}_{n} / \mathscr{F}_{n .5}$ has infinite rank was proved by Jiang in [Jia81] for $n=1$, by Cochran, Orr and Teichner in [COT04, Theorem 4.1] for $n=2$, and by Cochran, Harvey and Leidy in [CHL09, Theorem 8.1] for all $n$. Our main result is an analogous statement for the Grope filtration.

Theorem 5.2. For every $n \geq 2, \mathscr{E}_{n} / \mathscr{E}_{n .5}$ has infinite rank.

Our main result improves upon previous results regarding the non-triviality of the Grope filtration. For example, Cochran and Teichner proved in [CT07, Theorem 1.4] the existence of knots of infinite order in $\mathscr{E}_{n} / \mathscr{E}_{n .5}$. Since $\mathscr{E}_{n+2} \subset \mathcal{F}_{n}$ [COT03, Theorem 8.11], we are able to adapt Cochran, Harvey and Leidy's technique [CHL09] to prove that no non-trivial linear combination of these knots lies in $\mathcal{F}_{n .5}$, hence not in $\mathscr{E}_{n+2.5}$.

For the Grope filtration of the group of $m$-component string links with $m>$ 1, Harvey showed in [Har08, Theorem 6.13] that for all $n, \mathscr{E}_{n}^{m} / \mathscr{E}_{n+2}^{m}$ contains an infinitely generated subgroup. Let $\mathcal{B} \mathscr{E}_{n}^{m}$ denote the subgroup of $\mathscr{E}_{n}^{m}$ whose elements are boundary links. Combining [Har08, Theorem 6.13] and [CH08, Theorem 4.4], it is known that for each $m>1$ and $n \geq 2$, the abelianization of $\mathcal{B}_{n}^{m} / \mathscr{B} \mathscr{E}_{n+1.5}^{m}$ has infinite rank. We improve the result by narrowing the index gap, arriving at what should be the optimal result.

Theorem 6.2. For each $m>1$ and $n \geq 2$, the abelianization of $\mathscr{B}_{n}^{m} / \mathscr{B}_{n .5}^{m}$ has infinite rank; hence $\mathscr{E}_{n}^{m} / \mathscr{G}_{n .5}^{m}$ contains an infinitely generated subgroup.

\section{Definitions}

If $G$ is a group, the derived series of $G$ is defined recursively by $G^{(0)}=G$ and $G^{(i+1)}=\left[G^{(i)}, G^{(i)}\right]$. The rational derived series of $G$ is defined recursively by 
setting $G_{r}^{(0)}=G$ and

$$
G_{r}^{(i+1)}=\left\{g \in G: g^{k} \in\left[G_{r}^{(i)}, G_{r}^{(i)}\right], \text { for some } k>0\right\} .
$$

Let $M$ be closed, connected, orientable 3-manifold. Recall from [COT03] the definition of an $(n)$-solution for $M$.

Definition 2.1. A smooth, spin 4-manifold $W$ with $\partial W=M$ is an (n)-solution for $M$ if the inclusion-induced map $i_{*}: H_{1}(M) \rightarrow H_{1}(W)$ is an isomorphism and if there are embedded surfaces $L_{i}$ and $D_{i}$ (with product neighborhoods) for $i=1, \ldots, m$ that satisfy the following conditions:

(1) the homology classes $\left\{\left[L_{1}\right],\left[D_{1}\right], \ldots,\left[L_{m}\right],\left[D_{m}\right]\right\}$ form an ordered basis for $\mathrm{H}_{2}(\mathrm{~W})$,

(2) the intersection form $\left(H_{2}(W), \cdot\right)$ with respect to this ordered basis is a direct sum of hyperbolics,

(3) $L_{i} \cap D_{j}$ is empty if $i \neq j$,

(4) for each $i, L_{i}$ and $D_{i}$ intersect transversely at a point, and

(5) each $L_{i}$ and $D_{i}$ are $(n)$-surfaces, i.e., $\pi_{1}\left(L_{i}\right) \subset \pi_{1}(W)^{(n)}$ and $\pi_{1}\left(D_{i}\right) \subset$ $\pi_{1}(W)^{(n)}$.

If, in addition, $\pi_{1}\left(L_{i}\right) \subset \pi_{1}(W)^{(n+1)}$ for each $i$, we say $W$ is an (n.5)-solution for $M$.

There is a slight generalization of $n$-solvability, called rational $n$-solvability. For the purposes of this paper, it suffices to know that an $n$-solution is also a rational $n$ solution. If the above conditions hold except for the isomorphism on first homology, $W$ is called an (n)-bordism for $M$.

If a closed, orientable 3-manifold bounds an $(n)$-solution, we say $M$ is $(n)$ solvable. A knot $K$ in $S^{3}$ is said to be an $(n)$-solvable knot if 0 -surgery on $K$ is (n)-solvable.

As in [COT03], the set of all $(n)$-solvable knots is denoted $\mathscr{F}_{n}$, where it was shown that these $\mathscr{F}_{n}$ filter the topological knot concordance group $\mathcal{C}$, i.e., the $\mathscr{F}_{n}$ form a nested series of subgroups of $\mathcal{C}$ :

$$
\{0\} \subset \cdots \subset \mathscr{F}_{n .5} \subset \mathscr{F}_{n} \subset \cdots \subset \mathscr{F}_{1.5} \subset \mathscr{F}_{1} \subset \mathscr{F}_{0.5} \subset \mathscr{F}_{0} \subset \mathcal{C} .
$$

The following definition is modified from the definition in [FT95]:

Definition 2.2. A grope is a special pair (2-complex, base circle). A grope has a height $n \in \frac{1}{2} \mathbb{N}$. A grope of height 1 is precisely a compact, oriented surface $\Sigma$ with a single boundary component (the base circle). For $n \in \mathbb{N}$, a grope of height $n+1$ is defined recursively as follows: let $\left\{\alpha_{i}, \beta_{i}: i=1, \ldots, g\right\}$ be a symplectic basis of 
curves for $\Sigma$, the first stage of the grope. Here $g$ is the genus of $\Sigma$. Then a grope of height $n+1$ is formed by attaching gropes of height $n$ to each $\alpha_{i}$ and $\beta_{i}$ along the base circles.

A grope of height 1.5 is formed by attaching gropes of height 1 (i.e. surfaces) to a Lagrangian of a symplectic basis of curves for $\Sigma$. That is, a grope of height 1.5 is a surface with surfaces glued to 'half' of the basis curves (i.e. the $\alpha_{i}$ ). In general, a grope of height $n+1.5$ is obtained by attaching gropes of height $n$ to the $\alpha_{i}$ and gropes of height $n+1$ to the $\beta_{i}$.

Given a 4-manifold $W$ with boundary $M$ and a framed circle $\gamma \subset M$, we say that $\gamma$ bounds a Grope (note the capital ' $G$ ') in $W$ if $\gamma$ extends to a smooth embedding of a grope with its untwisted framing. That is, parallel push-offs of Gropes can be taken in $W$. Knots in $S^{3}$ are always equipped with the zero framing.

Cochran, Orr, and Teichner [COT03] also defined the Grope filtration of $\mathcal{C}$. The set of all knots that bound Gropes of height $n$ in $D^{4}$ is denoted $\mathscr{E}_{n}$, which is a subgroup of $\mathcal{C}$. Observe the following connection between gropes and the derived series: if a curve $\ell$ bounds a (map of a) grope of height $n$ in a space $X$, then $[\ell] \in \pi_{1}(X)^{(n)}$. It was shown in [COT03, Theorem 8.11] that $\mathscr{E}_{n+2} \subset \mathcal{F}_{n}$ (for all $n \in \frac{1}{2} \mathbb{N}$ ).

We will also use a few definitions from [CT07].

Definition 2.3. An annular grope of height $n$ is a grope of height $n$ that has an extra boundary component on its first stage. We say that the two boundary components of an annular grope cobound an annular grope. Two knots $K_{0}$ and $K_{1}$ are height $n$ Grope concordant if they cobound a height $n$ annular Grope $G$ in $S^{3} \times[0,1]$ such that $G \cap\left(S^{3} \times\{i\}\right)=K_{i}$ for $i=0,1$. For example, if $K$ is height $n$ Grope concordant to a slice knot, then $K \in \mathcal{E}_{n}$. The capital ' $\mathrm{G}$ ' in 'annular Grope' indicates the untwisted framing.

Let $G$ be a height $n$ Grope in $S^{3} \times[0,1]$ bounded by a knot $K \subset S^{3} \times\{0\}$. The union of sets of basis curves for each $n$-th stage surface of $G$ is called a set of tips for $G$. If the tips lie on annuli in $S^{3} \times[0,1]$ that are disjoint from each other and disjoint from the Grope (except at the tips) and the other boundary components of the annuli lie in $S^{3} \times\{1\}$, we say 'the tips are isotopic' to the link determined by the other boundary components of these annuli (a link in $S^{3} \times\{1\}$ ).

A capped Grope is the union of a Grope and a disjoint union of discs where the boundaries of the discs form a full set of tips of the Grope. The interiors of these discs must not intersect the Grope except perhaps on one of the boundary components of the Grope's first stage. These discs are called the 'caps' of the Grope. For example, if $K$ bounds a capped Grope, the caps are allowed to hit $K$.

The construction of our examples relies on a technique known as infection. Let $R$ be a fixed knot or link and $K$ be a fixed knot in $S^{3}$. Suppose $\alpha$ is a simple closed 
curve in $S^{3}-R$ such that $\alpha$ is itself the unknot. Some number $m$ of strands of $R$ pierce the disc bounded by $\alpha$. Let $R(\alpha, K)$ denote the knot obtained by replacing the $m$ trivial strands of $R$ by $m$ strands 'tied into the knot $K$.' See Figure 1. More precisely, one obtains $R(\alpha, K)$ by removing a regular neighborhood of $\alpha$ and gluing in $S^{3}-K$ in such a way that identifies the meridian of $\alpha$ with the longitude of $K$ and the longitude of $\alpha$ with the meridian of $K$. We say $R(\alpha, K)$ is the result of infecting $R$ by $K$ along $\alpha$.

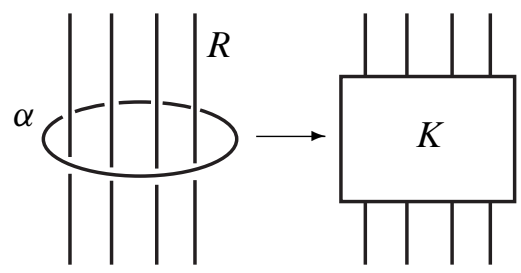

Figure 1 . Infecting $R$ by $K$ along $\alpha$.

Let $(H, J)$ denote the zero-framed, ordered Hopf link in the complement of a knot or link $R$ in Figure 2. Surgery on $(H, J)$ is homeomorphic to $S^{3}$ [Kir78, $\S 4$, Proposition 2], so the image of $R$ after performing this Hopf surgery is a new knot or link in $S^{3}$. By sliding the strands of $R$ (that pass through $J$ ) over $H$ and cancelling the Hopf pair, one sees the effect of Hopf surgery in Figure 2. We will use the terminology 'performing the handle cancellation $J-H$ ' to mean 'performing $(H, J)$ Hopf surgery.' This convention matches [CT07, p. 350].

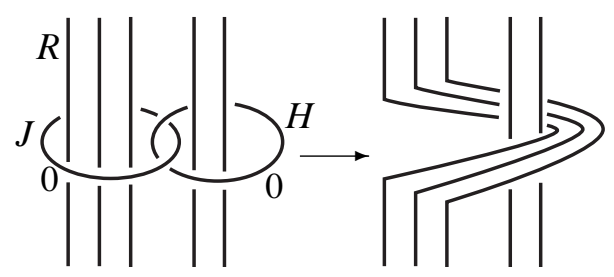

Figure 2. The effect of a Hopf surgery.

\section{Knots bounding Gropes of height $n+2$}

Fix an $m \geq 1$. For each $n \geq 1$, we will construct a knot $K_{m}^{n}$ by infecting a seed knot $R$ along certain curves in $\pi_{1}\left(S^{3}-R\right)^{(1)}$ by a knot $K_{m}^{n-1}$. Roughly speaking, we construct a height $n+2$ Grope bounded by $K_{m}^{n}$ in two stages. First we prove each 
$K_{m}^{n-1}$ bounds a height $n+1$ Grope whose tips are isotopic to meridians of $K_{m}^{n-1}$. When we infect $R$ by $K_{m}^{n-1}$, we will see that the tips of this Grope are isotopic to the infection curves, which we will then prove bound capped Gropes of height 1 . Gluing these height 1 capped Gropes onto the tips of the height $n+1$ Gropes for $K_{m}^{n-1}$ (and gluing a few annuli and slice discs) will result in a height $n+2$ Grope bounded by $K_{m}^{n}$.

3.1. The infecting knots $\boldsymbol{P}_{\boldsymbol{m}}$. Let $P_{\boldsymbol{m}}$ be the knot defined in Figure 3. A priori, we have described $P_{m}$ as a knot in some closed, oriented 3-manifold that is the image of $U$ after performing 0-surgery on the $(10+4 m)$-component link in Figure 3. Using Kirby's calculus of framed links (see, for example, [GS99, Chapter 5]), one can check that this 3-manifold is $S^{3}$.

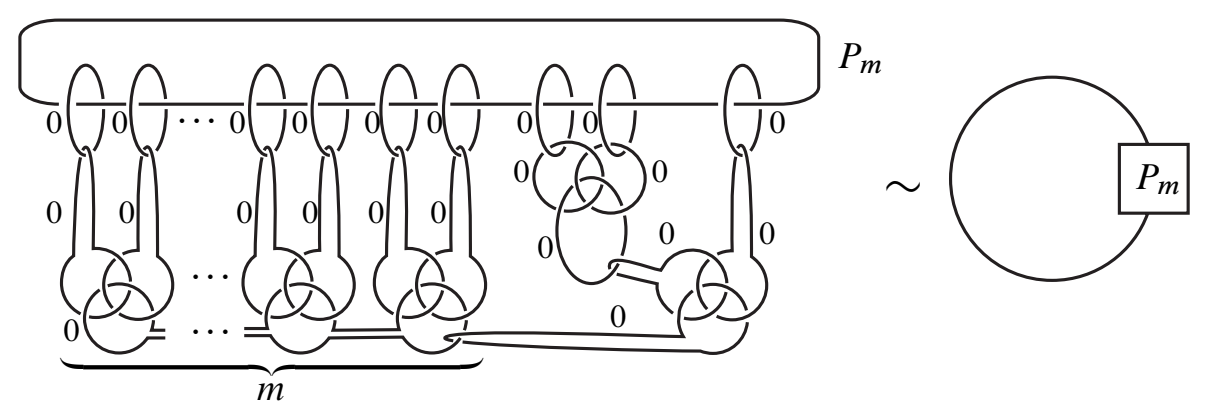

Figure 3. A surgery description of $P_{m}$.

Proposition 3.1. Each $P_{m}$ cobounds with the unknot a height 2 annular Grope in $S^{3} \times[0,1]$ whose tips are isotopic to a set of disjoint copies of the meridian of $P_{m}$.

Proof. We claim that Figure 5 depicts a height 2 annular grope in $S^{3}$ between $P_{m}$ and $U$. Let $L$ denote the $(10+4 m)$-component link $L=\left(H_{0}, \ldots, H_{9+4 m}\right)$ in Figure 4; the link $L$ is isotopic to the link defining $P_{m}$ in Figure 3. In Figure 4, we have shaded in some punctured surfaces bounded by the components $H_{0}, H_{4}$, and $H_{9}$. Once we perform 0 -surgery on $L$, these punctured surfaces will become closed surfaces, and these surfaces will be used to construct a height 2 Grope.

We perform $2 m+2$ handle cancellations,

$$
\begin{array}{r}
H_{7}-H_{5}, \quad H_{8}-H_{6}, \quad H_{12}-H_{10}, \quad H_{13}-H_{11}, \\
\ldots, H_{8+4 m}-H_{6+4 m}, \quad H_{9+4 m}-H_{7+4 m},
\end{array}
$$

to arrive at Figure 5 (ignore the $U$ and shaded parts for now). What remains is a 6-component link $N=\left(H_{0}, H_{1}, H_{2}, H_{3}, H_{4}, H_{9}\right)$. Let $N^{\prime}$ denote the link $N-$ 


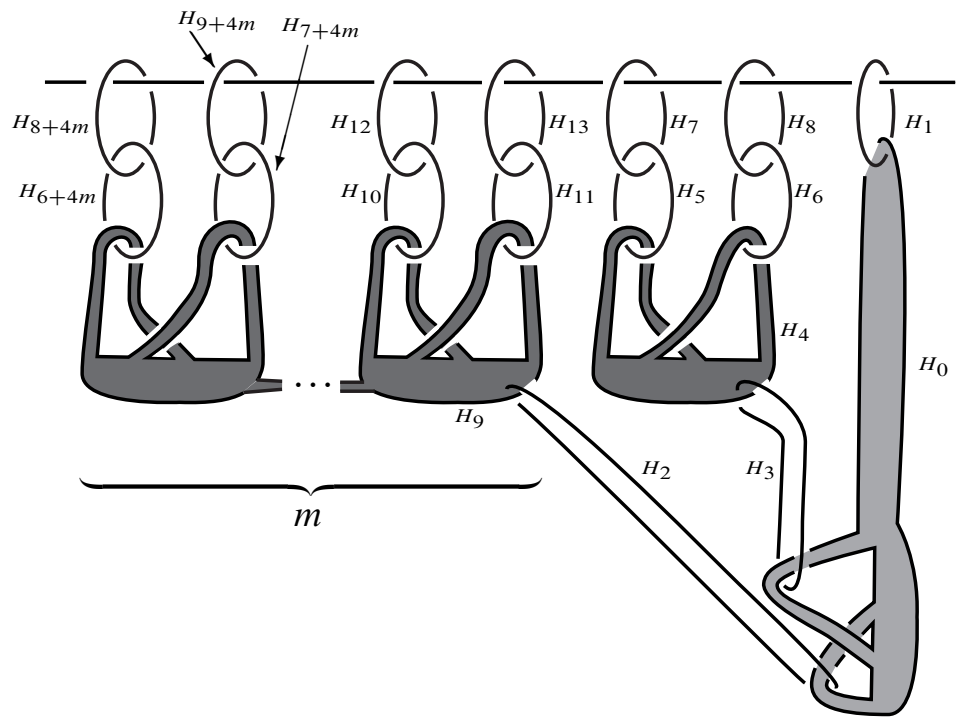

Figure 4. The link $L$.

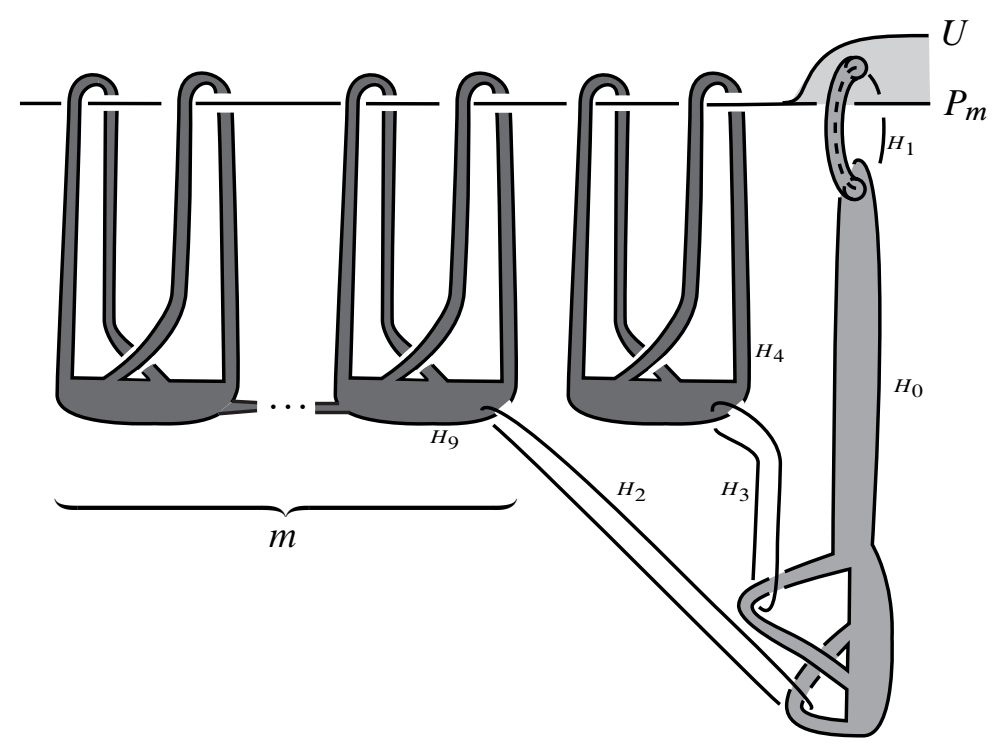

Figure 5. A height 2 Grope concordance from the unknot $U$ to $P_{m}$. 
$\left\{H_{0}, H_{1}\right\} ; N^{\prime}$ consists of two separated Hopf links, thus 0-surgery on $N^{\prime}$ (denoted $S_{N^{\prime}}^{3}$ ) is homeomorphic to $S^{3}$. Let $U$ be the knot in Figure 5 that deviates only slightly from $P_{m}$. The resulting knot $U^{\prime}$ after 0 -surgery on $N^{\prime}$ is the unknot in $S^{3} \cong S_{N^{\prime}}^{3}$, since $U$ does not hit the discs bounded by $H_{1}, H_{2}$ and $H_{3}$.

There is a punctured annulus in $S^{3}$ bounded by $U$ and $P_{m}$; the lightest shade of grey in Figure 5 is part of this annulus. The other part of this annulus has been collapsed to simplify the diagram. To the puncture, glue one boundary component of a tube. Push this annulus over $H_{1}$, and glue the other boundary component to the circle where $H_{1}$ punctures the torus on which $H_{0}$ lies (second darkest shade of grey in Figure 5). So far we have a height 1 (and genus 1) annular grope between $P_{m}$ and the unknot in $S^{3}$.

One may take the obvious symplectic basis elements for the aforementioned torus, tube them over $\mathrm{H}_{2}$ and $\mathrm{H}_{3}$ and attach them to punctured the surfaces for $\mathrm{H}_{4}$ and $\mathrm{H}_{9}$ (the darkest shade of grey in Figure 5). Recall that after zero surgery on the link, $H_{4}$ and $H_{9}$ lie on closed surfaces. Now we have a height 2 annular grope in $S^{3}$ between $P_{m}$ and the unknot. Figure 5 shows that the tips of this annular grope are isotopic to meridians of $P_{m}$.

One can push the $U$ boundary of component of this annular grope in the positive $I$ direction and the $P_{m}$ boundary component in the negative $I$ direction to arrive at the desired result.

3.2. The seed knot $\boldsymbol{R}$ and the infection curves. Our examples are inspired by Cochran, Harvey and Leidy's family $J_{n}$. They construct their family inductively by infecting the $9_{46}$ knot along the curves $\alpha$ and $\beta$ in Figure 6 . Note that the obvious tori
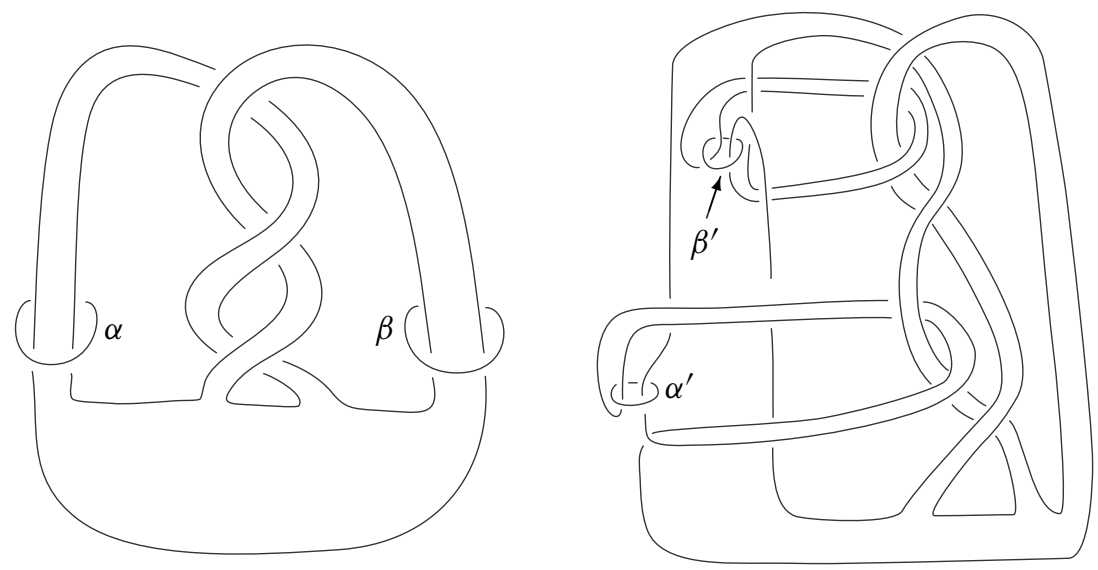

Figure 6. The infection curves $\alpha$ and $\beta$, and homotopic infection curves $\alpha^{\prime}$ and $\beta^{\prime}$. 
in $S^{3}-9_{46}$ bounded by $\alpha$ and $\beta$ intersect. For our construction of the Gropes for $K_{m}^{n}$, it is necessary that our infection curves bound disjoint surfaces in the complement of $9_{46}$. We homotope $\alpha$ and $\beta$ to arrive at different infection curves $\alpha^{\prime}$ and $\beta^{\prime}$.

Let $R=9_{46}$. By Figure 7, the curves $\alpha^{\prime}$ and $\beta^{\prime}$ bound tori that are disjoint and that miss $R$. The torus bounded by $\alpha^{\prime}$ has a symplectic basis $x_{1}, x_{2}$ (the curve $x_{2}$ has been isotoped off of the torus) of curves that inherit the zero framing from the torus. The torus bounded by $\beta^{\prime}$ has a similar symplectic basis $y_{1}, y_{2}$. It is clear from Figure 7 that the curves $x_{1}, x_{2}, y_{1}, y_{2}$ bound disjoint discs that hit the knot $R$ transversely. Thus, $\alpha^{\prime}$ and $\beta^{\prime}$ bound disjoint capped tori in the complement of $R$.

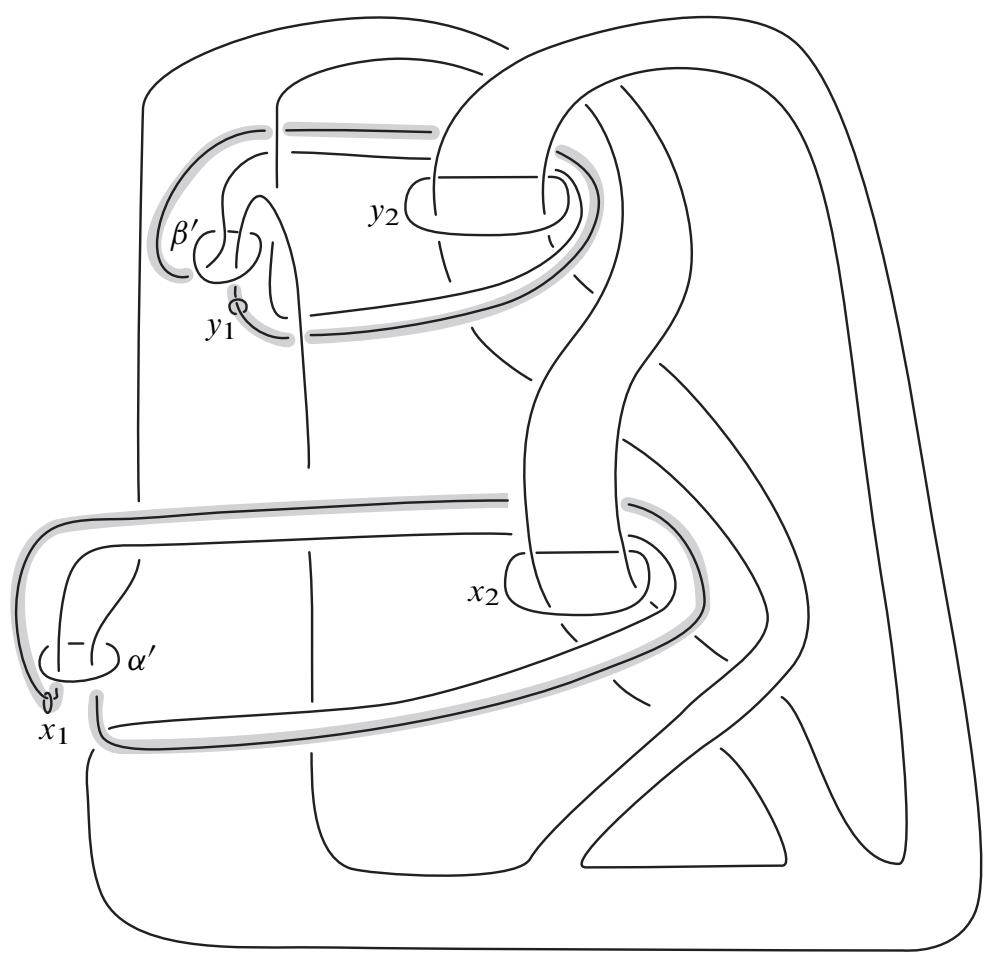

Figure 7. Infection curves bound disjoint capped tori.

3.3. Knots bounding Gropes of height $\boldsymbol{n}+2$. We now describe our family of knots $K_{m}^{n}$ for $n, m \geq 1$ with the property that $K_{m}^{n} \in \mathcal{E}_{n+2}$. Recall $R=9_{46}$, and $\alpha^{\prime}$ and $\beta^{\prime}$ be from Figure 6 . The curves $\alpha^{\prime}$ and $\beta^{\prime}$ bound punctured tori that are disjointly embedded in $S^{3}-R$. Let $K_{m}^{1}$ denote $R$ infected along $\alpha^{\prime}$ and $\beta^{\prime}$ by our knot $P_{m}$. 
Define $K_{m}^{n+1}$ by infecting $R$ along $\alpha^{\prime}$ and $\beta^{\prime}$ by $K_{m}^{n}$. Observe that $g\left(K_{m}^{n}\right) \leq 3$ since $\alpha^{\prime}$ and $\beta^{\prime}$ miss a genus three Seifert surface for $R$.

Proposition 3.2. Let $R$ be a knot in $S^{3}, \alpha$ an unknotted curve in $S^{3}-R$. If $K$ and $J$ are knots that are height $n$ Grope concordant, then the results of infection $R(\alpha, K)$ and $R(\alpha, J)$ are height $n$ Grope concordant.

Sketch of Proof. The knots $R(\alpha, K)$ and $R(\alpha, J)$ differ by what happens inside a fixed ball $D^{3}$ :

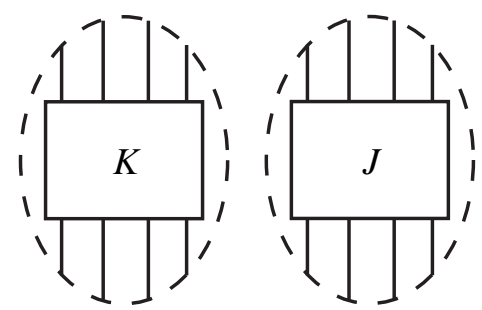

Let $\left(D^{3}, m K\right)$ denote the left-hand side of this diagram, i.e., a 3-ball with $m$ arcs tied into $K$. Since $K$ and $J$ are height $n$ Grope concordant, so are $\left(D^{3}, m K\right)$ and $\left(D^{3}, m J\right)$. We see this by slitting a height $n$ Grope concordance from $K$ to $J$, which turns it into a 'height $n$ slit Grope concordance' from one knotted arc to another. Taking parallel copies of this slit Grope concordance in $D^{3} \times[0,1]$ yields $G$, the 'height $n$ slit Grope concordance' in $D^{3} \times[0,1]$ from $\left(D^{3}, m K\right) \times\{0\}$ to $\left(D^{3}, m J\right) \times\{1\}$. We simply glue this $G$ to $\left(R-\left(R \cap D^{3}\right)\right) \times[0,1]$ to arrive at a height $n$ Grope concordance from $R(\alpha, K)$ to $R(\alpha, J)$.

Proposition 3.3. Let $R=9_{46}$ and $K_{m}^{1}$ be defined as above. Then $K_{m}^{1}$ is height 3 capped Grope concordant to $R$.

Proof. For simplicity, let $K=K_{m}^{1}$. $K$ is $R$ infected along $\alpha^{\prime}$ and $\beta^{\prime}$ by $P_{m}$. We will first concentrate on the infection along the $\alpha^{\prime}$ curve. Let $K^{\prime}$ denote $R$ infected along $\alpha^{\prime}$ by $P_{m}$. We will infect along $\beta^{\prime}$ later, arriving at $K$. Consider a diagram for the link $R, \alpha^{\prime}$. The knot $K^{\prime}$ can be described by replacing the infection curve with the framed $(10+4 m)$-component link that defines $P_{m}$. Figure 8 depicts this operation.

One may form a new knot $L$ by pulling the 2 strands of $K^{\prime}$ out of the upper-rightmost 0-framed curve in Figure 8. This is an '2-stranded analogue' of what is done in Figure 5. As in Proposition 3.1, there is a punctured annulus in $S^{3}$ between $K^{\prime}$ and $L$ (it is punctured twice). These punctures are 2 parallel copies of a meridian of the 0 -framed curve through which we pulled the 2 strands of $K^{\prime}$. Float these punctures downward in the $I$ direction so that each puncture is in a different $S^{3} \times\{*\}$ level. To each puncture, one can glue the height 2 Grope for $P_{m}$ described in Proposition 3.1. As before, the tips of the Grope in the $S^{3} \times\{*\}$ level are isotopic to parallel copies 


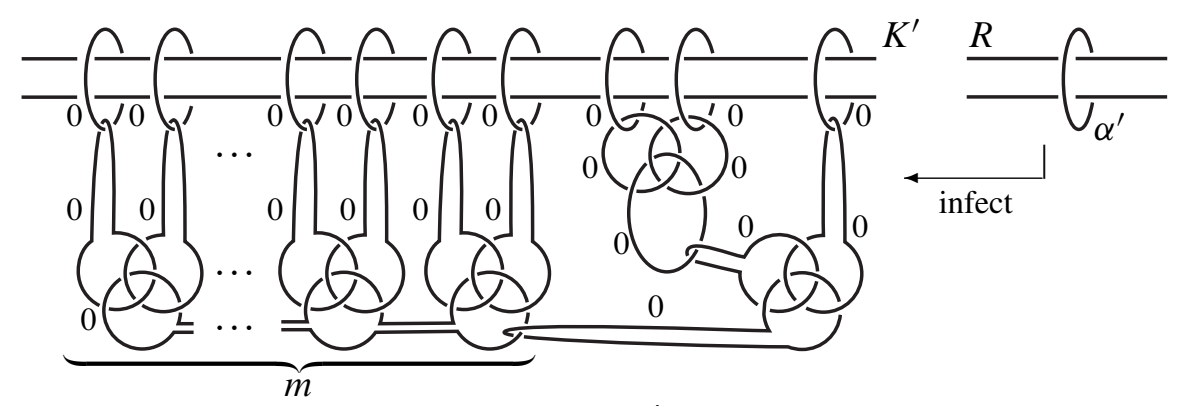

Figure 8. The knot $K^{\prime}$ as infection on $R$.

of the infection curve $\alpha^{\prime} \times\{*\}$. Now, we may float each of these $\alpha^{\prime} \times\{*\}$ disjointly down to the $S^{3} \times\{1\}$ level. Since each of the pieces of the Grope (and the tips) miss $K^{\prime}$ and $L$, we may glue $L \times[0,1]$ to this Grope. The result is a height 2 Grope concordance from $K^{\prime} \times\{0\}$ to $L \times\{1\}$ whose tips are disjointly isotopic to parallel copies of $\alpha^{\prime} \times\{1\}$.

We claim that $L$ is in fact $R$. The proof of this fact is similar to the proof that the $U$ from Figure 5 and Proposition 3.1 is the unknot. Namely, we have expressed $L$ as surgery on separated Hopf links, and for each of these Hopf pairs, $L$ does not pass through one of the components. Thus, performing the surgery does not change $L$ locally, and away from the surgery link, $L$ is identically $R$.

At this point we have proven $K^{\prime}=R\left(\alpha^{\prime}, P_{m}\right)$ is height 2 Grope concordant to $R$, and the tips of this Grope concordance (which we name $G$ ) are isotopic to parallel copies of $\alpha^{\prime}$. We can view $K$ as $K^{\prime}\left(\beta^{\prime}, P_{m}\right)$. An argument similar to the one above yields a height 2 Grope concordance (which we name $H$ ) between $K$ and $K^{\prime}$ whose tips are isotopic to parallel copies of $\beta^{\prime}$. We can glue $H \subset S^{3} \times[0,1]$ to $G \subset S^{3} \times[1,2]$ and float the tips of $H$ down through $S^{3} \times[1,2]$ so that they miss $G$. In order to justify this, we recall from the proof of Proposition 3.2 that $G$ was constructed by gluing pieces of $R \times I$ (which miss $\beta^{\prime}$ ) to something that lived in $D^{3} \times I$, where $\alpha^{\prime} \subset D^{3}$ and $\beta^{\prime}$ was contained in $S^{3}-D^{3}$. Thus, $G \cap\left(\beta^{\prime} \times[1,2]\right)=\emptyset$. We see that $J:=G \cup H \cup\left(\beta^{\prime} \times[1,2] \sqcup \cdots \sqcup \beta^{\prime} \times[1,2]\right)$ is a height 2 Grope concordance between $K_{m}^{1}$ and $R$ with punctured caps, and these punctures are parallel copies of $\beta^{\prime}$ union parallel copies of $\alpha^{\prime}$ such that the punctures lie in $\left(S^{3}-R\right) \times\{2\}$. The result is a height 2 Grope concordance between $K=K_{m}^{1}$ and $R$ whose tips are the union of parallel copies of $\alpha^{\prime}$ and parallel copies of $\beta^{\prime}$. A schematic of $J$ is depicted in Figure 9, with $n=0$. Pushing further down into $S^{3} \times[2,3]$, we can glue parallel copies of the capped tori to the copies $\alpha^{\prime}$ and $\beta^{\prime}$. The reader will recall that these capped tori miss $R$. Since we attached a capped torus to each tip of $J$, we are left with a height 3 capped Grope concordance between $K_{m}^{1}$ and $R$, as desired. 
Theorem 3.4. For each $n, m \geq 1, K_{m}^{n}$ is height $n+2$ capped Grope concordant to $R=9_{46}$. In particular, $K_{m}^{n}$ bounds a Grope of height $n+2$ in $D^{4}$.

Proof. We induct on $n$. The claim is true in the case $n=1$ by Proposition 3.3. Assume that the claim is true for $K_{m}^{n}$.

By definition $K_{m}^{n+1}$ is $R$ infected along $\alpha^{\prime}$ and $\beta^{\prime}$ by $K_{m}^{n}$. As in the proof of Proposition 3.3, we first concentrate on the infection along $\alpha^{\prime}$. Let $K^{\prime}=R\left(\alpha^{\prime}, K_{m}^{n}\right)$. By the inductive hypothesis on $K_{m}^{n}$ and by Proposition 3.2, $K^{\prime}$ and $R=R(\alpha$, unknot) are height $n+2$ Grope concordant. This Grope concordance is built using the height $n+2$ capped Grope concordance $L$ between $K_{m}^{n}$ and $R$. Each cap of $L$ hits $R$ transversely, and removing neighborhoods of the intersection points from the cap yields a planar surface with boundary equal to a tip of $L$ and copies of the meridian of $R$. The meridians of $R$ can float along $L$ to be meridians of $K_{m}^{n}$. Under the infection of $R$ along $\alpha^{\prime}$ by $K_{m}^{n}$, the meridian of $K_{m}^{n}$ is identified with (the longitude of a regular neighborhood of) $\alpha^{\prime}$. Let $G$ denote the union of $L$ and these planar surfaces reaching out from the tips. By the 'punctures of $G$,' we mean the punctures of these punctured caps, each of which is a copy of $\alpha^{\prime}$.

As for the infection along $\alpha^{\prime}$, between $K_{m}^{n+1}=K^{\prime}\left(\beta^{\prime}, K_{m}^{n}\right)$ and $K^{\prime}$ there is a height $n+2$ Grope concordance $H$ with punctured caps whose punctures are copies of $\beta^{\prime}$. Since infecting along $\beta^{\prime}$ is a local operation near $\beta^{\prime}, H$ misses $\alpha^{\prime} \times[0,1]$.

We now describe the height $n+3$ capped Grope concordance between $K_{m}^{n+1}$ and $R$. Figure 9 shows a schematic of the ultimate Grope. We start with the height $n+2$ Grope concordance $H$ between $K_{m}^{n+1}$ and $K^{\prime}$. Imagine that $H$ is embedded in $S^{3} \times[0,1]$ so that $H \cap S^{3} \times\{0\}=K_{m}^{n+1}$ and $H \cap S^{3} \times\{1\}=K^{\prime}$. The punctures of $H$ are parallel copies of $\beta^{\prime}$ in $S^{3} \times\{1\}$. Now imagine that $G$, which is the Grope

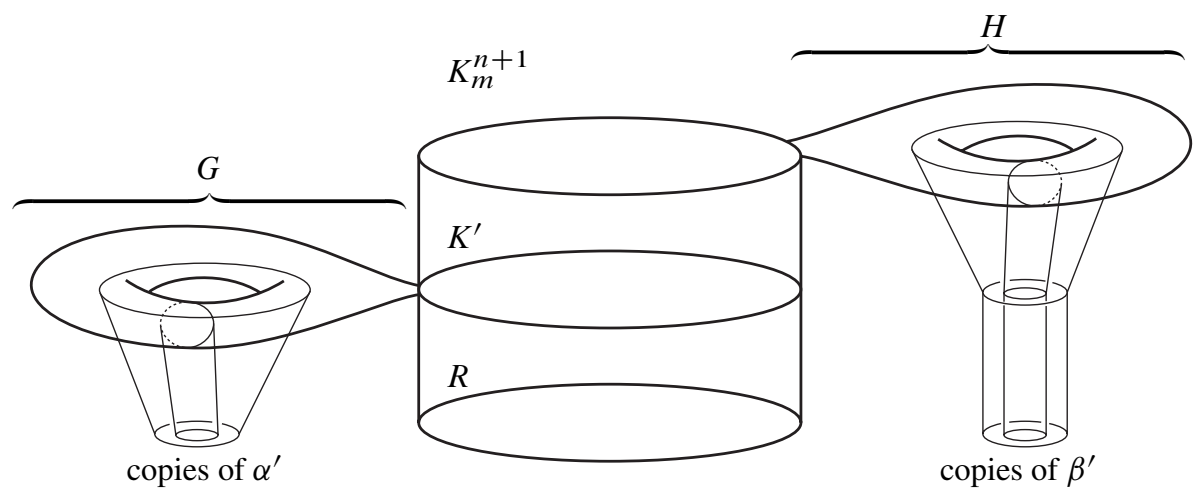

Figure 9. A schematic of the height $n+2$ Grope concordance, $J$, from $K_{m}^{n+1}$ to $R$. 
concordance between $K^{\prime}$ and $R$, is imbedded in $S^{3} \times[1,2]$ so that $G \cap S^{3} \times\{1\}=K^{\prime}$ and $G \cap S^{3} \times\{2\}=R$. The punctures of $G$ are parallel copies of $\alpha^{\prime}$ in $S^{3} \times\{2\}$. We claim that we can 'float down the punctures of $H^{\prime}$ ' into the $S^{3} \times\{2\}$ level while missing $G$. A justification of this was given in the proof of Proposition 3.3. We see that $J:=G \cup H \cup\left(\beta^{\prime} \times[1,2] \sqcup \cdots \sqcup \beta^{\prime} \times[1,2]\right)$ is a height $n+2$ Grope concordance between $K_{m}^{n+1}$ and $R$ with punctured caps, and these punctures are parallel copies of $\beta^{\prime}$ union parallel copies of $\alpha^{\prime}$ such that the punctures lie in $\left(S^{3}-R\right) \times\{2\}$.

Since $\alpha^{\prime}$ and $\beta^{\prime}$ bound disjoint capped torus in $S^{3}-R$, we can glue parallel copies of the capped torus (in $S^{3} \times[2,3]$ ) to $J$ along the punctures of $J$. To summarize, we have attached capped surfaces to each tip in the height $n+2$ Grope concordance $J$. What we have is a height $n+3$ capped Grope concordance between $K_{m}^{n+1}$ and $R$. We have proven the inductive step, and the claim is true for all $n$.

At this point, we have constructed an infinite family of knots in $\boldsymbol{E}_{n+2}$ for each $n \geq 0$. Before we show that each family generates an infinite-rank subgroup of $\mathscr{E}_{n+2} / \mathscr{E}_{n+2.5}$, we compute the classical signatures of our infecting knots $P_{m}$.

\section{Caclulation of $\rho$-invariants}

We now calculate the classical signatures of the $P_{m}$ and discuss why they are linearly independent. The linear independence of these invariants will ultimately guarantee that no linear combination of the $K_{m}^{n}$ lies in $\mathscr{Y}_{n+2.5}$. A proof of this will be outlined in Section 5.

Given a knot $P$, a Seifert matrix $V$ for $P$, and a unit complex number $\omega$ (which is not a root of the Alexander polynomial of $P$ ), one may form the Hermitian matrix $(1-\omega) V+(1-\bar{\omega}) V^{T}$. Let $\sigma_{P}(\omega)$ denote the signature of this matrix. This function is called the Levine-Tristram signature function of $P$ and does not depend on the choice of the Seifert matrix $V$. This function $\sigma_{P}: S^{1} \subset \mathbb{C} \rightarrow \mathbb{Z}$ is locally constant away from roots of the Alexander polynomial of $P$. Let $\rho_{0}(P)$ denote the average of $\sigma_{P}$ over $S^{1}$, i.e.,

$$
\rho_{0}(P)=\int_{S^{1}} \sigma_{P}(\omega) d \omega \in \mathbb{R}
$$

where the circle is normalized to have unit length. We call $\rho_{0}(P)$ the classical signature of $P$.

Lemma 4.1. The classical signature of $P_{m}$ is given by $\rho_{0}\left(P_{m}\right)=2-2 \frac{\theta_{m}}{\pi}$ where $\theta_{m}$ is the number satisfying $\cos \left(\theta_{m}\right)=\frac{2 \sqrt[3]{m}-1}{2 \sqrt[3]{m}}$ and $0<\theta_{m}<\pi$.

Proof. Let $E_{m}$ denote the exterior of $P_{m}$. Recall that $P_{m}$ is defined by surgery on the link in Figures 3 and 4. Depicted in Figure 10 is that link, isotoped for convenience. 


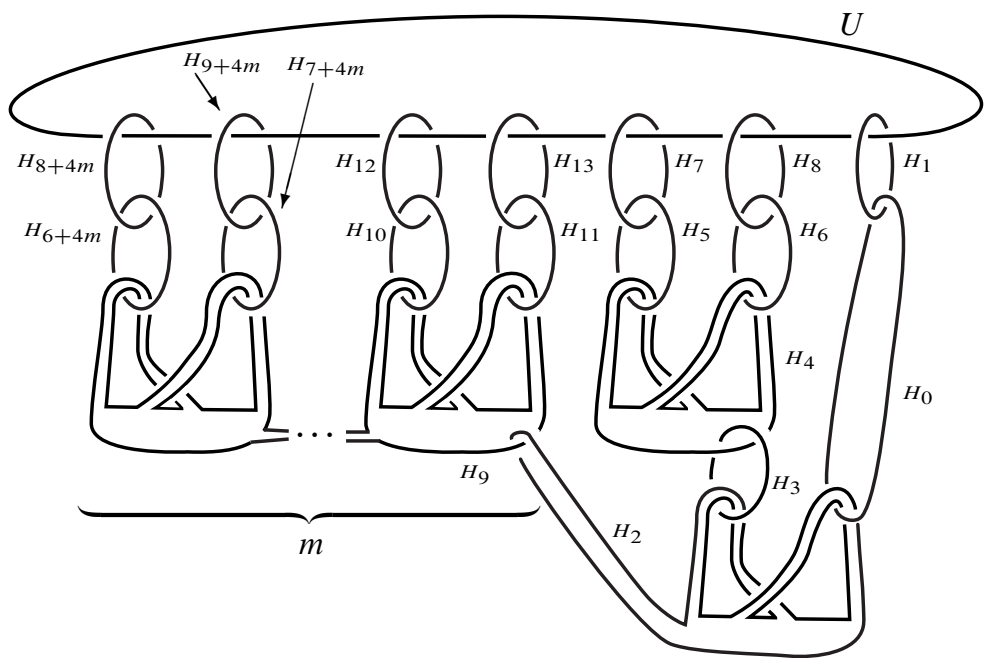

Figure 10. The surgery link defining $P_{m}$.

After performing the handle cancellations

$$
\begin{aligned}
H_{1}-H_{0}, & H_{7}-H_{5}, \quad H_{8}-H_{6}, \quad H_{12}-H_{10}, \\
H_{13}-H_{11}, & H_{8+4 m}-H_{6+4 m}, \quad H_{9+4 m}-H_{7+4 m},
\end{aligned}
$$

we arrive at the link in Figure 11. Thus, we can describe $E_{m}$ as the image of $S^{3}-U$ after 0 -surgery on the two component link $\left\{H_{9}, H_{2}\right\}$ as in Figure 11. This link is the result after performing 0 -surgery on all components of the link in Figure 3 except for the bottom-most two. As discussed in the proof of Proposition 3.1, the link $\left\{H_{9}, H_{2}\right\}$ is a link in $S^{3}$.

As in [Rol76, §7.C], we can compute the Alexander module of $E_{m}$ using these surgery instructions. Let $\alpha$ denote a lift of $H_{9}$ in the infinite cyclic cover of $U$, and let $\beta$ denote the lift of $\mathrm{H}_{2}$ that links $\alpha$ once. Let $\tau$ denote a generator of the group of covering translations. Recall that the matrix

$$
\lambda=\left(\begin{array}{cc}
a+\sum_{i \in \mathbb{Z}-\{0\}} \operatorname{lk}\left(\alpha, \tau^{i} \alpha\right) t^{i} & \sum_{i \in \mathbb{Z}} \operatorname{lk}\left(\alpha, \tau^{i} \beta\right) t^{i} \\
\sum_{i \in \mathbb{Z}} \operatorname{lk}\left(\beta, \tau^{i} \alpha\right) t^{i} & b+\sum_{i \in \mathbb{Z}-\{0\}} \operatorname{lk}\left(\beta, \tau^{i} \beta\right) t^{i}
\end{array}\right)
$$

where $a+\sum_{i \in \mathbb{Z}-\{0\}} 1 \mathrm{k}\left(\alpha, \tau^{i} \alpha\right)$ equals the framing of $H_{9}$ and $b+\sum_{i \in \mathbb{Z}-\{0\}} \operatorname{lk}\left(\beta, \tau^{i} \beta\right)$ equals the framing of $H_{2}$, is a presentation matrix for the Alexander module of $E_{m}$.

Displayed in Figure 12 is the link $\left\{U, H_{9}, H_{2}\right\}$ after an isotopy. Figure 13 depicts the infinite cyclic cover of $E_{m}$. 


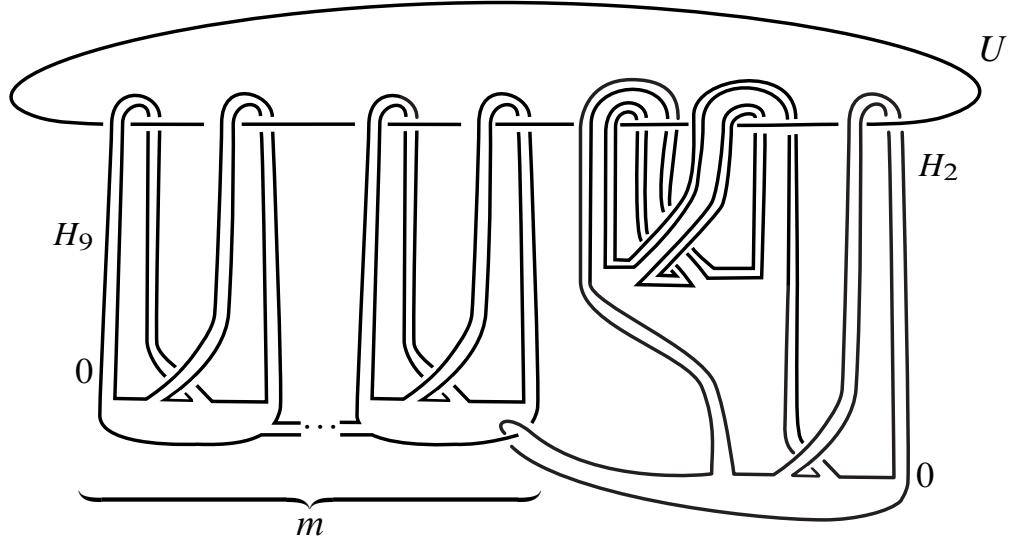

Figure 11. Zero surgery on the link $\left\{H_{2}, H_{9}\right\}$ yields $E_{m}$.

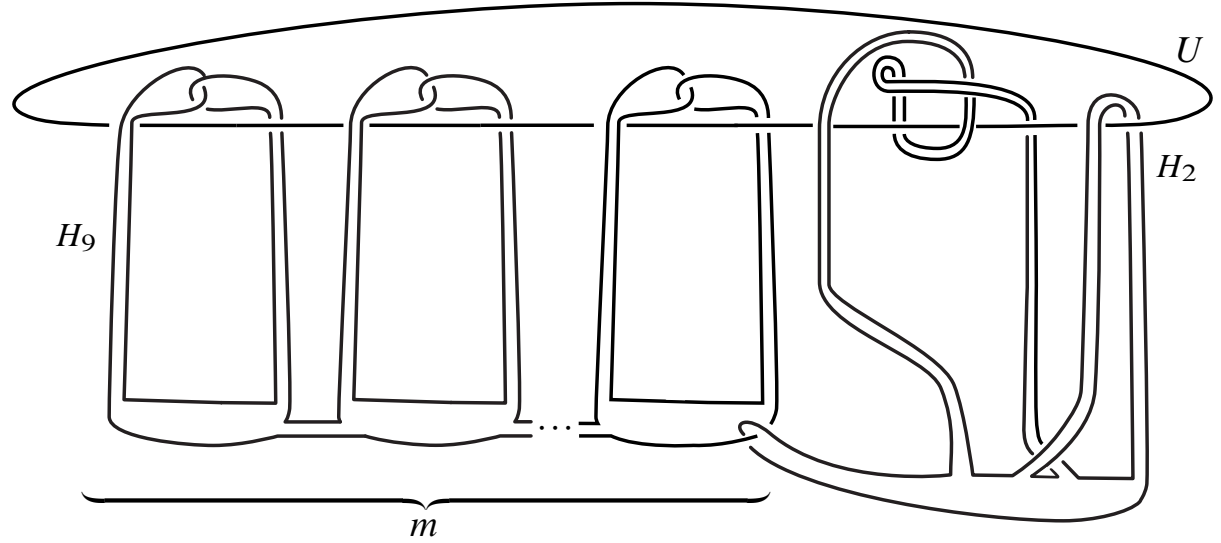

Figure 12. An isotopic image of the link from Figure 11.

From Figure 13, one computes the Alexander matrix to be

$$
\lambda=\left(\begin{array}{cc}
m\left(2-t-t^{-1}\right) & 1 \\
1 & t^{2}+t^{-2}-4\left(t+t^{-1}\right)+6
\end{array}\right) .
$$

The Alexander polynomial of $P_{m}$ is

$$
\Delta_{m}(t)=\operatorname{det} \lambda=m\left(\left(t^{3}+t^{-3}\right)-6\left(t^{2}+t^{-2}\right)+15\left(t+t^{-1}\right)-\frac{20 m-1}{m}\right) .
$$

Recall that $\rho_{0}$ of a knot is equal to the integral of the Levine-Tristram signature function over the unit circle (normalized to have length one), and that this function 


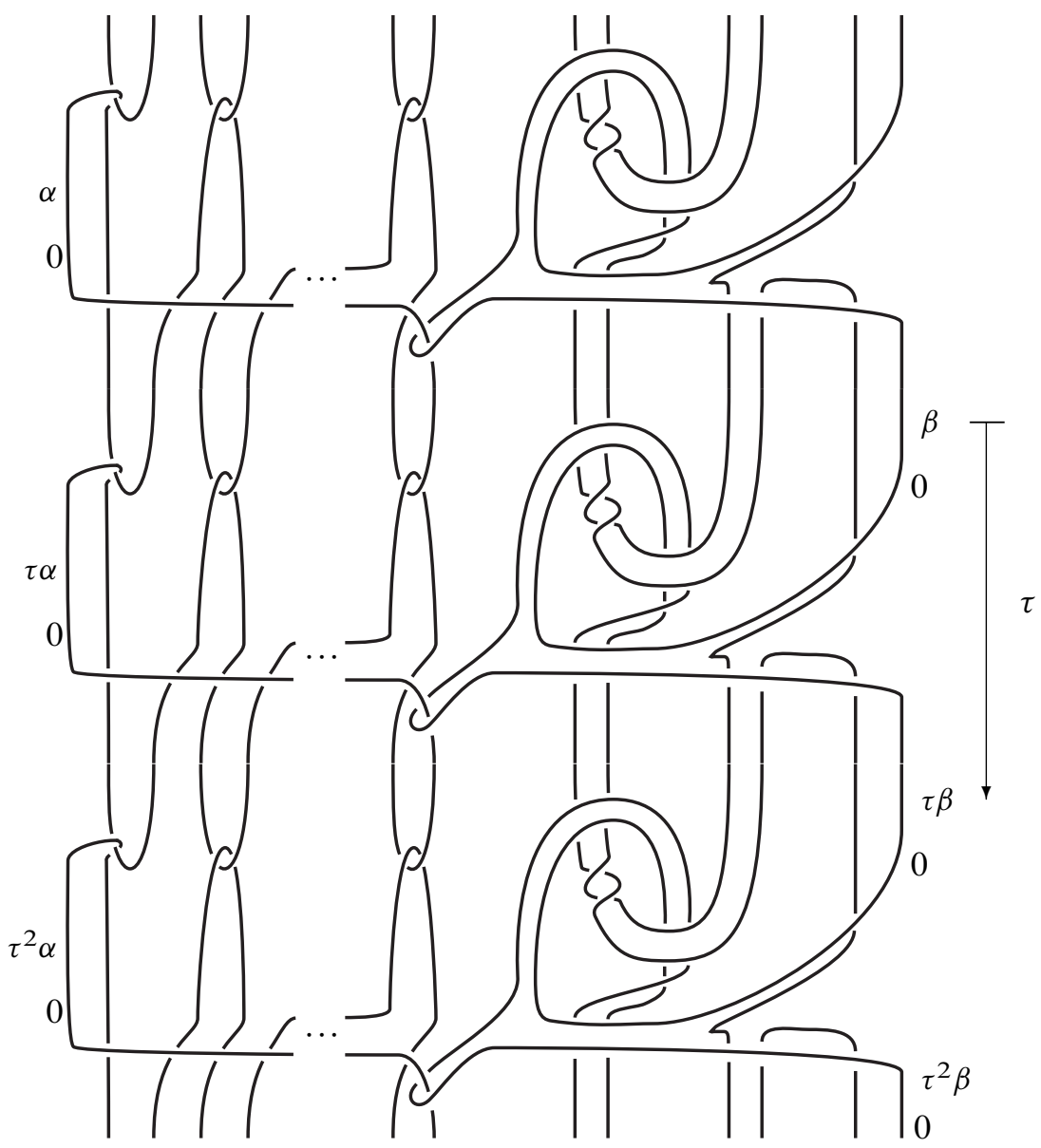

Figure 13. The infinite cyclic cover of $E_{m}$.

is constant away from the roots of the Alexander polynomial. We claim that the polynomial $\Delta_{m}(t)$ has exactly two roots on the unit circle, namely $e^{ \pm i \theta_{m}}$, where $\cos \left(\theta_{m}\right)=\frac{2 \sqrt[3]{m}-1}{2 \sqrt[3]{m}}$ and $0<\theta_{m}<\pi$. After proving this claim, we can conclude the signature of $\lambda$ is +2 when substituting $t=-1$, thus $\rho_{0}\left(P_{m}\right)=2-2 \frac{\theta_{m}}{\pi}$.

It is easy to verify that $e^{ \pm i \theta_{m}}$ are roots of $\Delta_{m}(t)$ and that $\Delta_{m}(t)$ factors as

$$
m\left(t-\frac{2 \sqrt[3]{m}-1}{\sqrt[3]{m}}+t^{-1}\right)\left(t^{2}+t^{-2}-\left(4+\frac{1}{\sqrt[3]{m}}\right)\left(t+t^{-1}\right)+6+\frac{2}{\sqrt[3]{m}}+\frac{1}{\sqrt[3]{m^{2}}}\right) .
$$

Assume that $e^{i \psi}$, where $\psi \in \mathbb{R}$, is a root of the second factor in Equation (1). 
Then $\psi$ must satisfy the equation

$$
4(\cos \psi)^{2}-\left(8+\frac{2}{\sqrt[3]{m}}\right) \cos \psi+4+\frac{1}{\sqrt[3]{m^{2}}}+\frac{2}{\sqrt[3]{m}}=0 .
$$

The quadratic formula yields $\cos \psi=\frac{4 \sqrt[3]{m}+1 \pm i \sqrt{3}}{4 \sqrt[3]{m}}$. This implies that $\psi \notin \mathbb{R}$, and we conclude that $\Delta_{m}(t)$ has no other roots on the unit circle.

Lemma 4.2. There is a subsequence of $P_{m_{j}}$ of the knots $P_{m}$ whose set of classical signatures is linearly independent over $\mathbb{Q}$.

Proof. By Lemma 4.1, it is sufficient to find a set $\left\{\theta_{m_{j}}\right\}_{j=1}^{\infty}$ that is linearly independent over $\mathbb{Q}$. Let $5=p_{1}<p_{2}<p_{3}<\cdots$ be a sequence of prime integers satisfying $p_{j} \equiv 1(\bmod 4)$ for all $j$, and set $m_{j}=\frac{1}{64}\left(p_{j}-1\right)^{6} \in \mathbb{Z}$. It was shown in [COT04, Proposition 2.6] that $\left\{\theta_{m_{j}}\right\}_{j=1}^{\infty}$ is linearly independent over $\mathbb{Q}$.

As a consequence of the additivity of the classical signature, we can immediately prove Theorem 5.2 for the case $n=0$.

Proof that $\mathscr{E}_{2} / \mathscr{E}_{2.5}$ has infinite rank. Proposition 3.1 implies that each $P_{m}$ bounds a Grope of height 2. The family $\left\{P_{m}\right\}$ generates an infinite-rank subgroup of $\mathscr{E}_{2} / \mathscr{E}_{2.5}$. By [COT03, Theorem 1.1], a knot lies in $\mathscr{E}_{2.5}$ if and only if it is algebraically slice. It is well-known that $\rho_{0}$ vanishes on algebraically slice knots and is additive under the connect sum operation. By the linear independence of $\left\{\rho_{0}\left(P_{m}\right)\right\}$ (Lemma 4.2) and these remarks, any non-trivial linear combination of the $P_{m}$ cannot lie in $\mathscr{E}_{2.5}$.

\section{Algebraic invariants}

We outline a proof that $K_{m}^{n}$ (or a subsequence of similar knots) are linearly independent in $\mathscr{E}_{n+2} / \mathscr{G}_{n+2.5}$. The following arguments are adapted from Cochran, Harvey and Leidy's proof that $\mathcal{F}_{n} / \mathcal{F}_{n .5}$ has infinite rank (cf. [CHL09, Theorem 8.1]).

Before the proof, a brief review of von Neumann $\rho$-invariants is in order. A more thorough treatment can be found in [CT07]. Given a closed 3-manifold and a homomorphism $\phi: \pi_{1}(M) \rightarrow \Gamma$ where $\Gamma$ is any group, one can define the von Neumann $\rho$-invariant $\rho(M, \phi) \in \mathbb{R}$. For a fixed $M$, Cheeger and Gromov have proven the existence of a constant $C_{M}$ such that $\left|\rho\left(M, \phi: \pi_{1}(M) \rightarrow \Gamma\right)\right|<C_{M}$ for all homomorphisms $\phi$ and groups $\Gamma$ [CG85].

Cochran, Orr and Teichner [COT03, Lemma 5.9] have shown that if $W$ is a 4manifold with $\partial W=M$, and if $\phi: \pi_{1}(M) \rightarrow \Gamma$ factors through $\bar{\phi}: \pi_{1}(W) \rightarrow \Gamma$, then

$$
\rho(M, \phi)=\sigma^{(2)}(W, \bar{\phi})-\sigma(W)
$$


where $\sigma^{(2)}(W, \bar{\phi})$ is the $L^{(2)}$-signature associated to $\bar{\phi}: \pi_{1}(W) \rightarrow \Gamma$ and $\sigma(W)$ is the ordinary signature of $W$. We refer the reader to [COT03, Section 5] for the rigorous definition of $\sigma^{(2)}(W, \bar{\phi})$. These von Neumann $\rho$-invariants are commonly referred to as 'signature defects.' Oftentimes one requires that $\Gamma$ be a poly-torsion-free-abelian group (PTFA). This acronym will appear in the sketch of the proof of our main result for the sake of correctness, but its precise definition is not crucial to understanding the main idea of the proof.

Definition 5.1. Let $K$ be a knot in $S^{3}$ and $G=\pi_{1}\left(M_{K}\right)$. The real number $\bar{\rho}(K)$ is the 'first-order signature' given by the von-Neumann $\rho$-invariant

$$
\rho\left(M_{K}, \phi: G \rightarrow G / G^{(2)}\right) .
$$

Theorem 5.2. For $n \geq 0, \mathscr{E}_{n+2} / \mathscr{E}_{n+2.5}$ has infinite rank.

Remark 1. As stated, our main result is not the strongest possible assertion. We produce an infinite family of knots in $\mathscr{E}_{n+2}$ whose members bound smooth embeddings of gropes. This family is a subsequence of $\left\{K_{m}^{n}\right\}_{m=1}^{\infty}$, or possibly a subsequence of a related family (see the proof below for a discussion). A result ([CHL09, Theorem 8.1]) of Cochran, Harvey and Leidy implies that no non-trivial linear combination of these knots is even topologically (n.5)-solvable, hence does not bound even a topologically flat embedding of a height $n+2.5$ grope in $D^{4}$. To avoid unnecessary reproduction of previous results, we will give a brief outline of the proof and refer the reader to [CHL09] for the details. We merely push through the proof given by Cochran, Harvey and Leidy, just to verify that their argument may be used to show that no non-trivial linear combination of the $K_{m}^{n}$ lie in $\mathscr{F}_{n .5}$, hence not in $\mathscr{E}_{n+2.5}$.

Sketch of proof for the case $n \geq 1$. First, we must find a genus one ribbon knot $R$ with $\bar{\rho}(R) \neq 0$. If $\bar{\rho}\left(9_{46}\right) \neq 0$, we can set $R=9_{46}$. Otherwise, we can tie one band of $9_{46}$ into the right-handed trefoil knot, as shown in Figure 14; the result, $R$, is a ribbon knot with $\bar{\rho}(R) \neq 0$.

Second, we must find an infinite set $\mathcal{P}$ of knots $P_{m}$ such that no non-trivial rational linear combination of $\left\{\rho_{0}\left(P_{m}\right)\right\}$ is a rational multiple of $\bar{\rho}(R)$. This family $\mathcal{P}$ is an infinite subset of the family $\left\{P_{m}\right\}$ defined in Section 3.1. These $\rho_{0}$-invariants are the classical signatures discussed in Section 4. Our Lemma 4.2 provided a subfamily of the $P_{m}$ with linear independent classical signatures, but now we require that $\left\{\rho_{0}\left(P_{m}\right)\right\} \cup\{\bar{\rho}(R)\}$ is linearly independent. Using elementary linear algebra, one can show that this set is linearly independent (after possibly taking a subsequence of $P_{m}$ ).

For each $n \geq 0$, we define a family of knots $\left\{K_{m}^{n}: m \geq 1\right\} \subset \mathscr{G}_{n+2}$. This family is defined as in Section 3.3, except using the knot $R$ as the seed knot. It should be noted that (possibly) having tied a trefoil in one of the bands does not affect the 


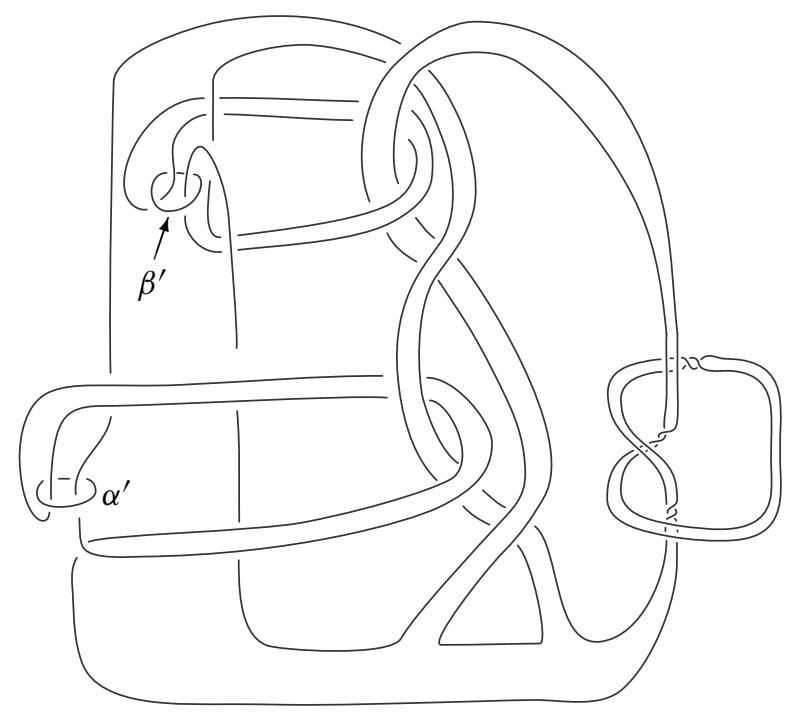

Figure 14. Tying one band of $9_{46}$ into a trefoil.

conclusions of any of the results in Section 3. In particular, these $K_{m}^{n}$ lie in $\mathscr{E}_{n+2}$. For notational simplicity, let $K_{m}^{0}=P_{m}$.

Now we must prove that no non-trivial linear combination of the knots $K_{m}^{n}$ is (even rationally) (n.5)-solvable. Since $\mathscr{E}_{n+2.5} \subset \mathscr{F}_{n .5}$, it will follow that no such combination of these knots lies in $\mathscr{\Theta}_{n+2.5}$, hence these knots are linearly independent in $\mathscr{E}_{n+2} / \mathscr{E}_{n+2.5}$. Suppose that a finite sum $\tilde{K}=\#_{m=1}^{\infty} j_{m} K_{m}^{n}$ is rationally $(n .5)$ solvable. We may assume that $j_{1} \geq 0$. We construct a family of 4-manifolds $W_{i}$ and reach a contradiction. Let us abbreviate $M_{K_{m}^{n}}=M_{m}^{n}$.

Consider $W_{n}$ with boundary $M_{1}^{0} \sqcup M_{1}^{0} \sqcup M_{R}$. Recall that $M_{1}^{0}=M_{K_{1}^{0}}=$ $M_{P_{1}}$. Let $\pi=\pi_{1}\left(W_{n}\right)$ and consider $\phi: \pi \rightarrow \pi / \pi_{r}^{(n+1)}$. Then by property (3) of Proposition 5.3,

$$
\rho\left(M_{P_{1}}, \phi_{\alpha^{\prime}}\right)+\rho\left(M_{P_{1}}, \phi_{\beta^{\prime}}\right)+\rho\left(M_{R}, \phi_{R}\right)=\rho\left(\partial W_{n}, \phi\right)=-\sum_{m} C_{m} \rho_{0}\left(P_{m}\right)
$$

where $C_{1} \geq 0$ and $\phi_{R}: \pi_{1}\left(M_{R}\right) \rightarrow \pi / \pi_{r}^{(n+1)}$ is induced by inclusion (and similarly for $\phi_{\alpha^{\prime}}$ and $\phi_{\beta^{\prime}}$ ). By property (2) of Proposition 5.3, $j_{*}\left(\pi_{1}\left(M_{P_{1}}\right)\right) \subset \pi^{(n)}$, implying that the restrictions $\phi_{\alpha^{\prime}}$ and $\phi_{\beta^{\prime}}$ factor through the respective abelianizations. Additionally by property (2), at least one of these coefficient systems is non-trivial. Hence, by Proposition 5.3,

$$
\rho\left(M_{P_{1}}, \phi_{\alpha^{\prime}}\right)+\rho\left(M_{P_{1}}, \phi_{\beta^{\prime}}\right)=\epsilon \rho_{0}\left(P_{1}\right)
$$


where $\epsilon$ is equal to one or two. Thus we can simplify equation 2 to yield

$$
\left(\epsilon+C_{1}\right) \rho_{0}\left(P_{1}\right)+\sum_{m>1} C_{m} \rho_{0}\left(P_{m}\right)=-\rho\left(M_{R}, \phi_{R}\right)
$$

where $\epsilon+C_{1} \geq 1$. Also by property (2) of Proposition 5.3,

$$
j_{*}\left(\pi_{1}\left(M_{R}\right)\right) \subset \pi^{(n-1)}
$$

so $\phi_{R}$ factors through $G / G^{(2)}$ where $G=\pi\left(M_{R}\right)$. Since $\operatorname{ker}\left(\phi_{R}\right) \subset G^{(1)}, \phi_{R}$ is determined by the kernel, denoted $A$, of the induced map

$$
\overline{\phi_{R}}: G^{(1)} / G^{(2)} \rightarrow \operatorname{im}\left(\phi_{R}\right) .
$$

$A$ is a submodule of the Alexander module $G^{(1)} / G^{(2)}$. Since the Alexander polynomial of $R$ is $(2 t-1)(t-2)$, the product of two irreducible coprime factors, the Alexander module of $R$ has only four submodules: the trivial submodule, $A$ itself, and $\left\langle\alpha^{\prime}\right\rangle$ and $\left\langle\beta^{\prime}\right\rangle$. One can examine these four possibilities for $A$ and conclude that $\rho\left(M_{R}, \phi_{R}\right) \in\{0, \bar{\rho}(R)\}$. Combining this with equation 3 , we have

$$
\left(\epsilon+C_{1}\right) \rho_{0}\left(P_{1}\right)+\sum_{m>1} C_{m} \rho_{0}\left(P_{m}\right)=C_{0} \bar{\rho}(R)
$$

where $C_{0} \in\{0,1\}$. Since $\epsilon+C_{1} \geq 1$, we have written a multiple of $\bar{\rho}(R)$ as a non-trivial linear combination of $\left\{\rho_{0}\left(P_{m}\right)\right\}$, a contradiction.

Proposition 5.3 (Proposition 8.2 of [CHL09]). If $\tilde{K}$ is rationally (n.5)-solvable, then for each $0 \leq i \leq n$, there is a 4-manifold $W_{i}$ with the following properties. Let $\pi=\pi_{1}\left(W_{i}\right)$.

(1) $W_{i}$ is a rational (n)-bordism where, for $i<n, \partial W_{i}=M_{1}^{n}$ and $\partial W_{n}=M_{1}^{0} \sqcup$ $M_{1}^{0} \sqcup M_{R}$;

(2) under the inclusion(s) $j: M_{1}^{n-i} \subset \partial W_{i} \rightarrow W_{i}$,

$$
j_{*}: \pi_{1}\left(M_{1}^{n-i}\right) \subset \pi^{(i)},
$$

and for each $i$ (at least one of the copies of ) $M_{1}^{n-i} \subset \partial W_{i}$,

$$
j_{*}:\left(\pi_{1}\left(M_{1}^{n-i}\right)\right) \cong \mathbb{Z} \subset \pi^{(i)} / \pi_{r}^{(i+1)}
$$

and under the inclusion $j: M_{R} \subset \partial W_{n} \rightarrow W_{n}$,

$$
j_{*}:\left(\pi_{1}\left(M_{R}\right)\right) \cong \mathbb{Z} \subset \pi^{(n-1)} / \pi_{r}^{(n)} ;
$$

(3) for any PTFA coefficient system $\phi: \pi_{1}\left(W_{i}\right) \rightarrow \Gamma$ with $\Gamma_{r}^{(n+1)}=1$,

$$
\rho\left(\partial W_{i}, \phi\right)=\sigma_{\Gamma}^{(2)}\left(W_{i, \phi}\right)-\sigma\left(W_{i}\right)=-\sum_{m} C_{m} \rho_{0}\left(P_{m}\right)
$$

for some integers $C_{m}$ (depending on $\phi$ ) with $C_{1} \geq 0$.

We refer the reader to [CHL09] for a proof of this proposition. 


\section{String links}

We now turn our attention to generalizing our main result to links. We follow the notation of [Har08]. Let $\mathcal{C}(m)$ denote the group of $m$-component string links modulo concordance (the group operation is concatenation). If $L \in \mathcal{L}(m)$, let $\hat{L}$ denote the closure of $L ; \hat{L}$ is obtained by attaching the $m$-component trivial string link to the ends of $L$ in the obvious way. Let $\mathscr{E}_{n}^{m}$ denote the subset of $\mathcal{C}(m)$ defined by $L \in \mathscr{E}_{n}^{m}$ if the components of $\widehat{L}$ bound disjoint Gropes of height $n$ in $D^{4}$. It can be shown that the Grope filtration of $\mathcal{C}(m)$

$$
0 \subset \cdots \subset \mathscr{H}_{n .5}^{m} \subset \mathscr{H}_{n}^{m} \subset \cdots \subset \mathcal{E}_{1.5}^{m} \subset \mathscr{H}_{1}^{m} \subset \mathcal{C}(m)
$$

is a filtration of $\mathscr{C}(\mathrm{m})$ by normal subgroups. If $\mathscr{B}(\mathrm{m})$ denotes the subgroup of $\mathcal{C}(\mathrm{m})$ consisting of boundary string links, we define $\mathcal{B}_{n}^{m}=\mathscr{E}_{n}^{m} \cap \mathcal{B}(m)$.

There is also the $(n)$-solvable filtration of $\mathscr{C}(m)$, whose definition we omit, that is related to the Grope filtration by $\mathcal{E}_{n+2}^{m} \subset \mathcal{F}_{n}^{m}$ for all $n \in \frac{1}{2} \mathbb{N}$. Let $\mathcal{B F}_{n}^{m}=$ $\mathcal{F}_{n}^{m} \cap \mathcal{B}(m)$.

Remark 2. For each $n \in \mathbb{Z}$, Harvey [Har08, Definition 3.11] defined a link invariant $\rho_{n}(L) \in \mathbb{R}$. If $L$ is a knot and $n=0$, then Harvey's $\rho_{0}(L)$ is equal to the classical signature discussed in Section 4. The salient features of these $\rho_{n}$ are listed below:

(1) [Har08, Corollary 6.7] For each $n \geq 0$ and $m \geq 1, \rho_{n}: \mathcal{B}(m) \rightarrow \mathbb{R}$ is a homomorphism,

(2) $\left[\mathrm{CH} 08\right.$, Theorem 4.4] $\rho_{n}(L)$ vanishes for all $L \in \mathcal{B}^{\mathcal{F}^{2}}{ }_{n .5}^{m}$.

(3) [Har08, Theorem 5.8] If $T$ is the identity element of $\mathcal{B}(m), \eta$ an unknotted circle in the complement of $T$ that lies in $\pi_{1}\left(D^{2} \times I-T\right)^{(n)}-\pi_{1}\left(D^{2} \times I-T\right)^{(n+1)}$, and $T(\eta, K)$ denotes $T$ infected along $\eta$ by the knot $K$, then $\rho_{n}(T(\eta, K))=\rho_{0}(K)$, where $\rho_{0}(K)$ is the classical signature discussed in Lemma 4.2.

Harvey proved that the Grope filtration of the string link concordance group is a non-trivial filtration.

Theorem 6.1 (6.13 of [Har08] and 4.4 of [CH08]). For each $n \geq 1$ and $m \geq 2$, the abelianization of $\mathcal{B}_{n}^{m} / \mathcal{B} \mathcal{E}_{n+1.5}^{m}$ has infinite rank; hence $\mathcal{B}_{n}^{m} / \mathcal{B}_{n+1.5}^{m}$ is an infinitely generated subgroup of $\mathscr{\Theta}_{n}^{m} / \mathscr{E}_{n+1.5}^{m}$.

With our knots $P_{m} \in \mathcal{E}_{2}$, we are able to close the index gap.

Theorem 6.2. For each $n \geq 2$ and $m \geq 2$, the abelianization of $\mathcal{B}_{n}^{m} / \mathcal{B}_{n+.5}^{m}$ has infinite rank; hence $\mathcal{B}_{n}^{m} / \mathscr{B} \mathscr{E}_{n .5}^{m}$ is an infinitely generated subgroup of $\mathscr{E}_{n}^{m} / \mathscr{E}_{n+.5}^{m}$. 
Proof. For $n=2$, let $L_{k}$ denote the $m$-component boundary string link whose closure is the union of the $(m-1)$-component trivial link and the knot $P_{k}$ as defined in Section 3.1. Since $P_{k} \in \mathcal{E}_{2}$, the components of the closure of $L_{k}$ bound disjoint Gropes of height $2, m-1$ of which are discs. Thus $L_{k} \in \mathcal{B}_{2}^{m}$. The linear independence of the $L_{k}$ in $\mathscr{B}_{2}^{m} / \mathscr{B} \mathscr{E}_{2.5}^{m}$ follows from the argument below when $n \geq 3$.

Assume $n \geq 1$. We prove the statement for $n+2$. Let $F$ denote the free group on $m$ generators, and $T$ be the trivial $m$-component string link, so that $F \cong \pi_{1}\left(D^{2} \times I-T\right)$. Pick an element $[\eta] \in F^{(n)}-F^{(n+1)}$. By [CT07, Lemma 3.9], we may pick an embedded curve $\eta$ in $D^{2} \times I-T$ that bounds a disk in $D^{2} \times I$ and that bounds a Grope of height $n$ in $D^{2} \times I-T$. Let $S_{\eta}=\left\{T\left(\eta, P_{k}\right): P_{k}\right.$ as defined in Section 3.1 $\}$ where $T(\eta, K)$ is defined by item 3 of Remark 2 . Since $\eta \in F^{(1)}$, there are disjoint surfaces bounded by the components of $T$ that miss the curve $\eta$. Thus each $L \in S_{\eta}$ is a boundary string link. Since $P_{k} \in \mathcal{E}_{2}$, we have that if $L \in S_{\eta}$, then $\widehat{L} \in \mathcal{E}_{n+2}^{m}$. Thus, $S_{\eta} \subset \mathscr{B}_{n+2}^{m}$.

By items 1 and 2 of Remark 2, $\rho_{n+2}: \mathscr{B}_{n+2}^{m} / \mathscr{B} \mathcal{E}_{n+2.5}^{m} \rightarrow \mathbb{R}$ is a well-defined homomorphism. By item 3 of Remark 2, the image of $S_{\eta}$ under this homomorphism is $\rho_{n+2}\left(S_{\eta}\right)=\left\{\rho_{0}\left(P_{k}\right)\right\}$, which contains a $\mathbb{Q}$-linearly independent subset of $\mathbb{R}$ by Lemma 4.2. The conclusion follows.

\section{References}

[CG85] J. Cheeger and M. Gromov, Bounds on the von Neumann dimension of $L^{2}$-cohomology and the Gauss-Bonnet theorem for open manifolds. J. Differential Geom. 21 (1985), 1-34. Zbl 0614.53034 MR 0806699

[CH08] T. Cochran and S. Harvey, Homology and derived series of groups II: Dwyer's theorem. Geom. Topol. 12 (2008), 199-232. Zbl 1163.20031 MR 2377249

[CHL09] T. Cochran, S. Harvey, and C. Leidy, Knot concordance and higher-order Blanchfield duality. Geom. Topol. 13 (2009), no. 3, 1419-1482. Zbl 1175.57004 MR 2496049

[COT03] T. Cochran, K. Orr, and P. Teichner, Knot concordance, Whitney towers and $L^{2}$ signatures. Ann. of Math. 157 (2003), 433-519. Zbl 1044.57001 MR 1973052

[COT04] T. Cochran, K. Orr, and P. Teichner, Structure in the classical knot concordance group. Comment. Math. Helv. 79 (2004), 105-123. Zbl 1061.57008 MR 2031301

[CT07] T. Cochran and P. Teichner, Knot concordance and von Neumann $\rho$-invariants. Duke Math. J. 137 (2007), no. 2, 337-379. Zbl 05152567 MR 2309149

[FT95] M. Freedman and P. Teichner, 4-manifold topology I: Subexponential groups. Invent. Math. 122 (1995), 509-529. Zbl 0857.57017 MR 1359602

[GS99] R. Gompf and A. Stipsicz, 4-manifolds and Kirby calculus. Grad. Stud. in Math. 20, Amer. Math. Soc., Providence, R.I., 1999. Zbl 0933.57020 MR 1707327

[Har08] S. Harvey, Homology cobordism invariants and the Cochran-Orr-Teichner filtration of the link concordance group. Geom. Topol. 12 (2008), 387-430. Zbl 1157.57006 MR 2390349 
Vol. 85 (2010) The non-triviality of the Grope filtrations of the concordance groups

[Jia81] B. Jiang, A simple proof that the concordance group of algebraically slice knots is infinitely generated. Proc. Amer. Math. Soc. 83 (1981), no. 1, 189-192. Zbl 0474.57004 MR 0620010

[Kir78] R. Kirby, A calculus for framed links in $S^{3}$. Invent. Math. 45 (1978), 35-56. Zbl 0377.55001 MR 0467753

[Rol76] D. Rolfsen, Knots and links. Publish or Perish, Berkeley, CA, 1976. Zbl 0339.55004 MR 0515288

Received June 16, 2008

Department of Mathematics, Columbia University, MC 4403, 2990 Broadway, New York, NY 10027, U.S.A.

E-mail:pdhorn@math.columbia.edu 